\title{
DEFORMACIONES ARTIFICIALES DE LA BÓVEDA DEL CRÁNEO EN EL DELTA DEL PARANÁ Y CUENCA INFERIOR DEL RÍO URUGUAY
}

\author{
ARTIFICIAL CRANIAL VAULT DEFORMATION IN THE PARANÁ RIVER DELTA \\ AND LOWER URUGUAY RIVER
}

\author{
Mariano Carlos Del Papa*, Velén Aimé Pennini', Delfina Bonilla \\ ${ }^{1}$ División Antropología. Facultad de Ciencias Naturales y Museo. Universidad Nacional de La Plata. La Plata. Argentina
}

PALABRAS CLAVE deformaciones artificiales del cráneo; delta del Paraná; tramo inferior del río Uruguay; Holoceno tardío

\begin{abstract}
RESUMEN En este trabajo se presentan los resultados obtenidos del análisis de la variabilidad morfológica de la bóveda del cráneo de muestras pertenecientes a poblaciones humanas que habitaron el delta del Paraná y la cuenca inferior del río Uruguay durante el Holoceno tardío, mediante el uso de técnicas de morfometría geométrica. En este sentido, el objetivo se está dirigido a presentar una primera aproximación a la problemática de las deformaciones artificiales de la bóveda del cráneo para las regiones anteriormente mencionadas. Se utilizaron técnicas de la morfometría geométrica y análisis estadísticos multivariados con el objetivo de cuantificar patrones de variación entre las deformaciones artificiales del cráneo, a fin de aportar, desde una nueva línea de análisis, a las investigaciones bioarqueológicas que se vienen desarrollando en el
\end{abstract}

área. Para cumplir con este objetivo se llevó a cabo el análisis de 65 cráneos (40 femeninos y 25 masculinos) procedentes de nueve sitios ubicados en diferentes sectores del litoral: delta inferior del río Paraná, delta superior del río Paraná y margen oeste de la cuenca inferior del río Uruguay. Los resultados obtenidos permiten, por un lado, posicionar en un esquema general las relaciones entre prácticas deformatorias y cronologías absolutas de las poblaciones del delta del río Paraná y cuenca inferior del río Uruguay, y realizar comparaciones con los esquemas propuestos para otras regiones de Argentina. Por el otro, los análisis permitieron establecer la existencia de tendencias geográficas y temporales en la práctica de deformación intencional de cráneos. Rev Arg Antrop Biol 20(2), 2018. doi:10.17139/raab.2018.0020.02.04

KEY WORDS cranial vault deformations; Parana delta; the lower basin of the Uruguay river; late Holocene

ABSTRACT In this article we present the results from the geometric morphometric analyses of morphological variability of the cranial vault in samples belonging to human populations that inhabited the Paraná river delta and the lower basin of the Uruguay river during the late Holocene. The main goal was to present a first approach to the problems related to artificial cranial deformations in the regions mentioned above. Geometric morphometric techniques and multivariate statistical procedures were used in order to quantify patterns of variation among artificial cranial deformations with the aim of contributing from a new line of evidence to the bioarchaeological research that is being carried out in the area. Sixty-five skulls (40 female and 25 male) from nine sites located in different coastal areas were analyzed: the lower and upper delta of the Paraná river and the west bank of the lower basin of the Uruguay river. The results obtained enabled us, on the one hand, to establish relationships between deformation practices and the absolute chronology of the populations of the Paraná river delta in an overall scheme, and to compare them with those proposed for other regions. On the other hand, we were able to establish the existence of geographical and temporal trends in cranial vault deformation practices. Rev Arg Antrop Biol 20(2), 2018. doi:10.17139/raab.2018.0020.02.04

Desde fines del siglo XIX el estudio de las modificaciones artificiales de la bóveda del cráneo, ha sido abordado para desarrollar diferentes aspectos tales como, el reconocimiento de la influencia de este fenómeno en la variación

Financiamiento: Sin financiamiento.

*Correspondencia a: Mariano Carlos Del Papa. División Antropología, Facultad de Ciencias Naturales y Museo. Paseo del Bosque s/n. La Plata (1900). Argentina. E-mail: mdelpapa@fcnym.unlp.edu.ar

Recibido 16 Agosto 2016; aceptado 25 Octubre 2017

doi:10.17139/raab.2018.0020.02.04 
fenotípica craneofacial (Cocilovo y Varela, 2010; Cocilovo, Varela y O’Brien, 2010; Del Papa y Perez, 2007; Dembo y Imbelloni, 1938; Imbelloni, 1924-1925; Perez, 2006), el estudio de los efectos de la deformación craneal de la bóveda sobre el crecimiento y desarrollo craneofacial (Frieß y Baylac, 2003; Richtsmeier, Cheverud y Buikstra, 1984), como así también los estudios referidos a la clasificación de las deformaciones craneales (Broca, 1879; DemboyImbelloni, 1938; Imbelloni, 1924-1925; Virchow, 1892).

Estas clasificaciones tradicionales fueron generadas principalmente por la agrupación de cráneos que presentan similitudes en la morfología externa de su bóveda (Imbelloni, 1924-1925). Debido a las técnicas morfoscópicas aplicadas y al enfoque tipológico que caracterizaba esta primera fase de la investigación en antropología (véase Sober, 1980), se introdujo un sesgo significativo en el análisis de la variación de la deformación artificial de la bóveda craneal, resultando en una discriminación muchas veces incorrecta de la variación morfológica (Konigsberg, Kohn y Cheverud, 1993). Durante las últimas décadas del siglo $\mathrm{XX}$, se ha comenzado a utilizar un conjunto de técnicas alternativas al enfoque tipologista $y$ a la morfometría tradicional (Rohlf y Marcus, 1993), y se caracteriza por (Bookstein, 1991). Estas nuevas técnicas, denominadas como morfometría geométrica, son adecuadas para el estudio estadístico de la variación y covariancia de la forma de una estructura (Rohlf, 1998). El avance fundamental de la morfometría geométrica con respecto a la aproximación tradicional, reside en el desarrollo de técnicas diseñadas para el análisis de datos de forma, a partir de una configuración completa de puntos que corresponden a las ubicaciones de los puntos de referencia morfológicos (landmarks) (Bookstein, 1991) y semilandmarks (Bookstein, 1997) y que resultan invariantes con respecto a los efectos de traslación, rotación y escala geométrica (Rohlf, 2000). En los últimos años, la morfometría geométrica comenzó a tener un rol preponderante en las investigaciones antropobiológicas. En el contexto del presente trabajo, éstas se orientaron al estudio de diferentes aspectos vinculados con la morfología craneofacial entre los que se destacan la com- prensión de la representación espacio temporal de las deformaciones artificiales de la bóveda del cráneo en las poblaciones humanas que habitaron el actual territorio de la República Argentina (Bernal, González, Pérez y Pucciarelli, 2008).

A la vez, resulta importante subrayar que en tanto sea entendido como una práctica cultural, la alteración en la forma de la bóveda craneana puede ser vista como no intencional o como intencional (Brothwell, 1981; Ubelaker, 1984). En el primer caso, reflejan los hábitos funcionales, como por ejemplo el uso de cunas rígidas durante la infancia (Baffi y Berón, 1992; Pérez, 2007). Mientras que, en el segundo caso, se reconoce su función como un indicador de la identidad del grupo (Blom, 1999; Torres-Rouff y Yablonsky, 2005), así como estratificación social o como evidencia de procesos poblacionales que implican migración, movilidad y/o dinámica de los sistemas de parentesco (Berón y Luna, 2009).

El estudio de la distribución geográfica y cronológica de las deformaciones de la bóveda del cráneo en Argentina tuvo un desarrollo diferencial en las distintas regiones. Para el norte de la Patagonia encontramos el trabajo pionero de Bórmida (1950, 1953-1954), a partir del cual se estableció una secuencia cronológica de los diferentes tipos de deformación craneana a partir de la posición estratigráfica de los individuos. Como resultado de este trabajo, el autor concluye que existe una secuencia temporal donde la variedad plano-lámbdica correspondería a los momentos más tardíos. En el sudeste de la región pampeana, la sistematización de las investigaciones ha permitido elaborar un esquema de variación temporal en el que encontramos deformaciones de tipo anular o pseudo-circular del tipo tabular erecto hace 3000 años AP, deformación tabular oblicua con una antigüedad de 2000 años AP y planolámbdicas entre 1500 y 400 años AP (Bernal et al.,2008). En el caso de la deformación tabular erecto plano-lámbdica, atribuidas al Holoceno tardío final, se verifica su presencia más allá de las regiones anteriormente mencionadas describiéndose para el sur de Mendoza (Della Negra, Novellino, Perez, Hajduk y Bernal, 2008), provincia de La Pampa (Baffi y Berón, 1992; Berón y Baffi, 2003; Berón y Luna, 2009) y 
centro de la provincia de Santiago del Estero (Drube, 2010).

Para el área correspondiente al Delta del Paraná, las primeras investigaciones que dan cuenta de la existencia de registro bioarqueológico se remontan a los trabajos de Ramón Lista (Lista, 1878) y Zeballos y Pico (1878). A partir de ese momento y durante todo el siglo XX, se realizaron caracterizaciones del registro bioarqueológico (Caggiano, 1983; Caggiano, Flores, Méndez y Salceda, 1978; Torres, 1911; Vignati, 1941; entre otros) de esta región. En relación con el problema de la ocupación humana de estos territorios y sobre la diversidad cultural encontrada, el antropólogo Fernando Gaspary, en su obra de 1950, realiza una pormenorizada descripción de los hallazgos arqueológicos y antropológicos del Cerro Grande de la Isla de los Marinos (Gaspari, 1950). El autor hace hincapié en la comparación entre las características físicas de los primeros habitantes realizadas por cronistas europeos y los datos somatométricos obtenidos por Torres, para las poblaciones arqueológicas del delta inferior del río Paraná y por D'Orbigny (1944) para grupos guaraníes. De los resultados obtenidos, concluye que tanto los pobladores del Cerro Grande (delta superior del río Paraná) como los descriptos por Torres (1911) para el delta inferior del río Paraná. Por otro lado, las clasificaciones tipológicas de las deformaciones artificiales observadas por el autor son designadas como del tipo plano-lámbdica tabular erecta (Imbelloni, 1924-1925) para Cerro Grande y menciona la inexistencia de deformaciones artificiales para las muestras del delta medio e inferior del río Paraná. Finalmente, incorpora las descripciones sobre las prácticas deformatorias realizadas por Imbelloni (19241925) para grupos guaraníes que habitaron esta región, a las que asigna como tabular oblicua.

Con respecto al curso inferior del río Uruguay, aunque los primeros antecedentes registrados sobre investigaciones bioarqueológicas se sitúan a finales del siglo XIX (Outes, 1912), durante el siglo XX se observa una marcada disminución de los mismos. Existen caracterizaciones del registro bioarqueológico de los sitios Estación de Puerto Landa (Torres 1911), Túmulo I de la Pesquería Nicolini y Túmulo de Lucuix (Greslebin, 1931), y de El Aserradero (Fortich Baca, 1977).
A partir del año 2012, se comenzaron a realizar estudios bioarqueológicos sistemáticos de los sitios de la margen oeste de la cuenca inferior del río Uruguay (departamento de Gualeguaychú, provincia de Entre Ríos). Estos materiales se encuentran almacenados en el Museo Arqueológico Manuel Almeida y son el producto de las tareas de excavación realizadas por el profesor Manuel Almeida, desde 1950 hasta los primeros años del siglo XXI (Almeida, 1983a, Almeida, 1983b; Castro y Del Papa, 2015). Sin embargo, cabe destacar que hasta el momento no se han realizado descripciones sobre la presencia de deformaciones artificiales para el área correspondiente a la cuenca inferior del río Uruguay.

En este contexto, el objetivo general de este trabajo es analizar la variabilidad morfológica del contorno en norma lateral de la bóveda del cráneo, correspondiente a muestras de grupos prehispánicos que habitaron el delta superior e inferior del río Paraná y el curso inferior del río Uruguay durante el Holoceno tardío, mediante el uso de técnicas de morfometría geométrica. Como objetivos específicos se plantean: (1) realizar la cuantificación de la morfología del contorno en norma lateral de la bóveda del cráneo en muestras provenientes del delta superior del río Paraná, delta inferior del río Paraná y curso inferior del río Uruguay y (2) identificar la probable presencia de diferentes tendencias en las modificaciones antrópicas deliberadas de la bóveda del cráneo en las muestras anteriormente mencionadas.

\section{MATERIAL Y MÉTODOS}

El conjunto óseo analizado corresponde a 65 individuos adultos, de los cuales 40 fueron determinados como femeninos y 25 como masculinos. Las determinaciones de sexo y edad fueron realizadas a partir de los caracteres morfológicos del cráneo, siguiendo criterios bioarqueológicos estándar (Buikstra y Ubelaker, 1994). Estos conjuntos de cráneos proceden de colecciones osteológicas formadas durante el siglo XX a partir del trabajo de campo en distintos sitios del noreste argentino por parte de investigadores y aficionados. Específicamente, se han consultado nueve colecciones provenientes de tres sectores del litoral (Fig. 1): 1- delta Inferior del río Paraná: sitios Túmulo I y II del Paraná Guazú, Túmulo I del Brazo Largo y 
Túmulo I del Brazo Gutiérrez (Torres, 1911); 2delta superior del río Paraná: sitio Cerro Grande de La Isla Los Marinos (Gaspary, 1950, Kozameh y Brunás, 2011) y 3- margen oeste del curso inferior del río Uruguay: sitios Cerro Lorenzo 2, sambaquí de Puerto Landa, La Correntina y Túmulo I La Argentina (Castro y Del Papa, 2015). Actualmente estas colecciones se encuentran bajo la custodia de diferentes instituciones. Las colecciones provenientes del delta inferior del río Paraná se hallan en la División Antropología del Museo de La Plata (UNLP). Las del delta superior en el Instituto de Antropología de la Facultad de Filosofía y Letras (UNL). Finalmente, las de la cuenca inferior del río Uruguay están alojadas en el Museo Arqueológico Manuel Almeida de Gualeguaychú (Entre Ríos).

A continuación, se hará una breve descripción de las características más sobresalientes de los si- tios arqueológicos de los que provienen las muestras analizadas.

Cuatro de las muestras estudiadas proceden del delta inferior del río Paraná. Los sitios Túmulo I y II del Paraná Guazú corresponden a montículos sobre albardón ubicados en la margen derecha del río Paraná Guazú, frente a las Islas Los Platos y La Paloma, respectivamente (Torres, 1911) (Fig. 1). Ambos sitios fueron inicialmente excavados por Torres en el año 1905. Las tareas de excavación continuaron 20 años más tarde en el Túmulo I a través de los trabajos de Lothrop, quien llamó al lugar El Cerrillo (Lothrop, 1932). Como resultado de todas las actividades de campo en ambos sitios se han recuperado una gran cantidad de restos de cerámica grabada y lisa, artefactos líticos, instrumentos óseos y restos faunísticos (Bonomo, 2013;

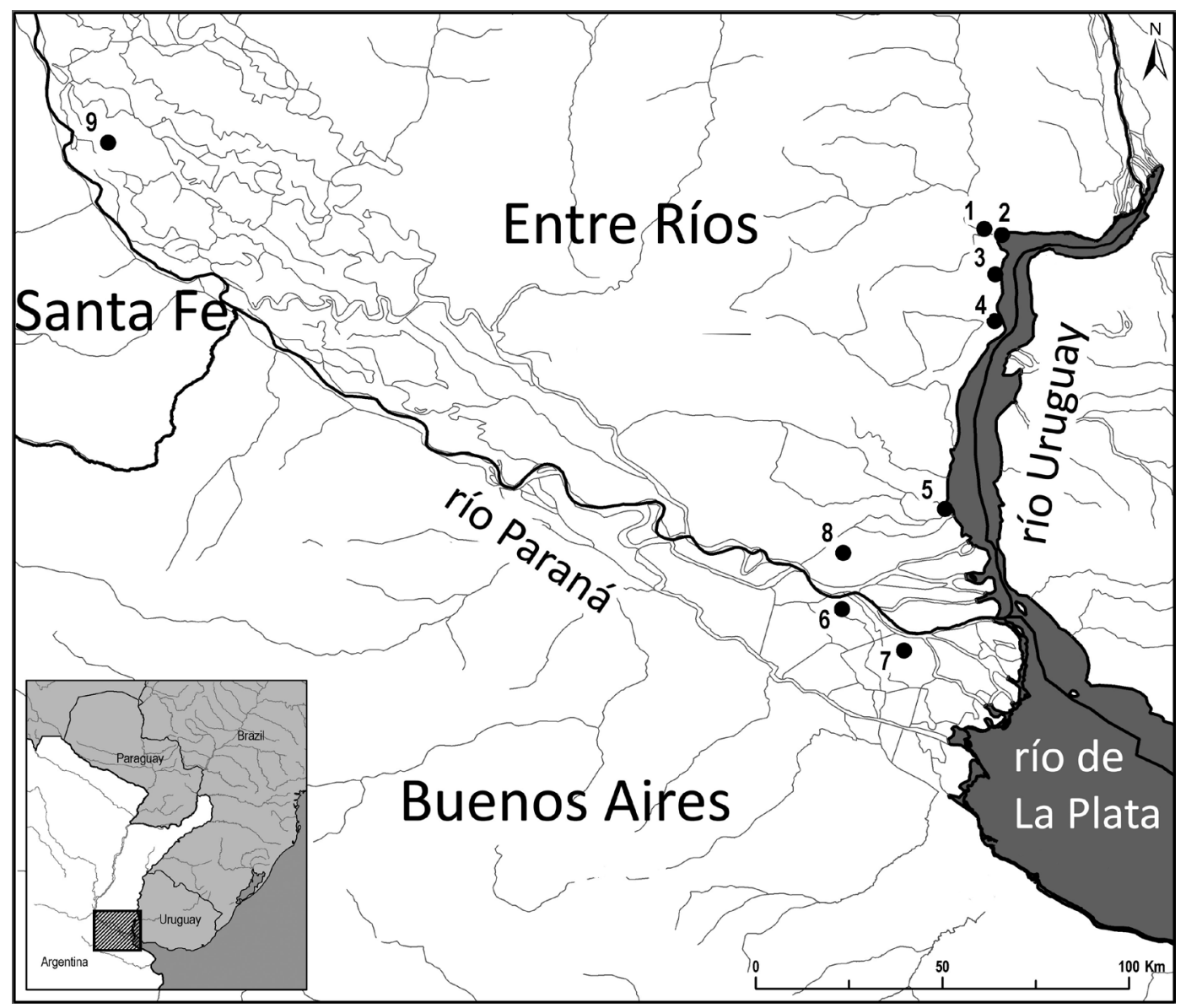

Fig. 1. Sitios correspondientes a las muestras en estudio. 1:La Correntina; 2:Cerro Lorenzo; 3:Túmulo I - La Argentina; 4: Sambaquí de Puerto Landa; 5:Túmulo I de Brazo Largo; 6:Túmulo I de Paraná Guazú; 7:Túmulo II de Paraná Guazú; 8:Túmulo I del Brazo Gutiérrez; 9:Cerro Grande de la Isla Los Marinos. 
Torres, 1911). También se encontró un considerable número de entierros humanos localizados sobre una de las laderas de los montículos: en el caso del Túmulo I, Torres halló más de 40 entierros y Lothrop, otros 23 (Lothrop, 1932; Ramos van Raap y Bonomo, 2016; Torres, 1911); mientras que en el Túmulo II, Torres describe el hallazgo de los esqueletos de más de 60 individuos, de los cuales extrajo solamente los elementos óseos de 39 (Torres, 1911). En ambos sitios, durante los trabajos de Torres, se hallaron láminas de cobre (caracterizadas como adornos personales) en las cercanías del cráneo de algunos individuos (Bonomo, Capdepont y Matarrese, 2009; Bonomo, Politis y Gianotti, 2011; Torres, 1911). Los fechados radiocarbónicos obtenidos sobre huesos humanos para estos sitios son de $576 \pm 42$ años AP para el Túmulo I y de $846 \pm 41$ años AP para el Túmulo II (Bernal, 2008; Bonomo et al., 2011) (Tabla 1). Para el es- tudio de las deformaciones craneanas se analizaron once cráneos, tres femeninos del Túmulo I y ocho cráneos (uno masculino y siete femeninos) procedentes del Túmulo II (Tabla 1).

El Túmulo I del Brazo Largo, al igual que los sitios anteriormente descriptos, es una estructura monticular ubicada en la margen izquierda del Brazo Largo, a $25 \mathrm{~km}$ de la desembocadura del río Uruguay (Fig. 1). Este sitio también fue excavado por Torres en 1906. Los hallazgos arqueológicos están constituidos por material lítico de diferentes materias primas, instrumentos óseos y fragmentos de cerámica grabada (Torres, 1911). Además, se suma el hallazgo de al menos seis entierros humanos, que según Torres corresponderían a cuatro individuos masculinos y a un femenino. Un fechado realizado sobre uno de los esqueletos arrojó una cronología de $656 \pm 42$ años AP (Bonomo et al., 2011). El análisis de la deformación craneana se realizó en un total de cua-

TABLA 1. Muestras utilizadas para el análisis de la variación morfológica en la bóveda craneal

\begin{tabular}{|c|c|c|c|c|c|c|}
\hline \multirow{2}{*}{ Procedencia } & \multicolumn{2}{|c|}{ Sexo } & \multirow{2}{*}{ Abr. } & \multirow{2}{*}{ Cronología } & \multirow{2}{*}{$\begin{array}{c}\text { Fechado } \\
\mathrm{C}^{14} \text { años AP }\end{array}$} & \multirow{2}{*}{ Referencia } \\
\hline & Masculino & Femenino & & & & \\
\hline $\begin{array}{l}\text { Túmulo I del } \\
\text { Paraná Guazú- } \\
\text { El Cerrillo }\end{array}$ & 0 & 3 & DI & $\begin{array}{l}\text { Holoceno } \\
\text { tardío final }\end{array}$ & $576 \pm 42$ & Bonomo et al., 2011 \\
\hline $\begin{array}{l}\text { Túmulo II del } \\
\text { Paraná Guazú }\end{array}$ & 1 & 7 & DI & $\begin{array}{l}\text { Holoceno } \\
\text { tardío final }\end{array}$ & $846 \pm 41$ & Bernal, 2008 \\
\hline $\begin{array}{c}\text { Túmulo I del Brazo } \\
\text { Largo }\end{array}$ & 0 & 4 & DI & $\begin{array}{l}\text { Holoceno } \\
\text { tardío final }\end{array}$ & $656 \pm 42$ & Bonomo et al., 2011 \\
\hline $\begin{array}{c}\text { Túmulo I del Brazo } \\
\text { Gutiérrez }\end{array}$ & 9 & 9 & DI & $\begin{array}{l}\text { Holoceno } \\
\text { tardío final }\end{array}$ & $752 \pm 41$ & Bernal, 2008 \\
\hline $\begin{array}{l}\mathrm{C}^{\circ} \text { Grande de la } \\
\text { Isla Los Marinos }\end{array}$ & 11 & 10 & DS & $\begin{array}{l}\text { Holoceno } \\
\text { tardío final }\end{array}$ & $\begin{array}{c}460 \pm 50 \\
590 \pm 60 \\
660 \pm 70\end{array}$ & $\begin{array}{c}\text { Kozameh y Brunás, } \\
2011\end{array}$ \\
\hline $\mathrm{C}^{\mathrm{o}}$ Lorenzo 2 & 3 & 5 & CiRU & $\begin{array}{l}\text { Holoceno } \\
\text { tardío inicial }\end{array}$ & $2050 \pm 60$ & $\begin{array}{c}\text { Castro y Del Papa, } \\
2015\end{array}$ \\
\hline $\begin{array}{l}\text { Sambaquí de } \\
\text { Puerto Landa }\end{array}$ & 0 & 1 & $\mathrm{CiRU}$ & $\begin{array}{l}\text { Holoceno } \\
\text { tardío final }\end{array}$ & $630 \pm 50$ & $\begin{array}{c}\text { Castro y Del Papa, } \\
2015\end{array}$ \\
\hline La Correntina & 0 & 1 & CiRU & $\mathrm{s} / \mathrm{f}$ & & $\begin{array}{c}\text { Castro y Del Papa, } \\
2015\end{array}$ \\
\hline $\begin{array}{c}\text { Túmulo I } \\
\text { La Argentina }\end{array}$ & 1 & 0 & CiRU & $\mathrm{s} / \mathrm{f}$ & & $\begin{array}{c}\text { Castro y Del Papa, } \\
2015\end{array}$ \\
\hline Total & 25 & 40 & & & & \\
\hline
\end{tabular}


tro cráneos, todos determinados por los autores como femeninos (Tabla 1).

El sitio Túmulo I del Brazo Gutiérrez se encuentra localizado en la margen norte del río Brazo Gutiérrez, a unos 700mts de la costa del mismo (Fig. 1). Este sitio fue excavado en 1906 por Torres (Torres, 1911) y como producto de las tareas de excavación se recuperaron varios restos arqueofaunísticos, instrumentos de hueso, fragmentos de cerámica y una lámina de cobre adherida a un cráneo humano (Torres, 1911). También se encontraron los esqueletos de más de 50 individuos, ubicados hacia la extremidad sur del montículo; sólo fueron exhumados los huesos de 33 individuos (todos adultos: 17 masculinos, 8 femeninos y 8 indeterminados). Se cuenta con un fechado radiocarbónico de $752 \pm 41$ años AP para las inhumaciones (Bernal, 2008). El análisis de la deformación craneana se realizó en18 cráneos, de los cuales nueve se determinaron como femeninos y nueve como masculinos (Tabla 1).

Del delta superior del río Paraná se analizaron los cráneos del sitio Cerro Grande de la Isla de Los Marinos. Los entierros humanos hallados aquí corresponden a los trabajos de excavación realizados por Gaspary durante 1947 (Gaspary, 1950). El sitio es una estructura monticular ubicada en el departamento Victoria (provincia de Entre Ríos), en la margen izquierda del río Paraná (Fig. 1) (Gaspary, 1950). Las excavaciones en este sitio permitieron detectar numerosos huesos humanos (correspondientes a un número mínimo de individuos $[(\mathrm{NMI})=48]$ de adultos y subadultos inhumados en entierros primarios, entierros secundarios en paquetes, huesos aislados, segmentos articulados y elementos calcinados. Recientemente, se realizaron tres fechados sobre la colección osteológica que arrojaron edades de $460 \pm 50,590 \pm 60$ y $660 \pm 70$ años AP (Kozameh y Brunás, 2013). El análisis de la deformación craneana se realizó en un total de 21 cráneos, de los cuales 10 se determinaron como femeninos y 11 como masculinos (Tabla 1).

La muestra proveniente de la cuenca inferior del río Uruguay está conformada por un conjunto de restos humanos hallados durante los trabajos llevados a cabo por el profesor Manuel Almeida y colaboradores entre 1955 y 2004. Durante las tareas de campo, realizadas principalmente en el departamento de Gualeguaychú (provincia de Entre Ríos), se detectaron 12 sitios con entierros humanos (Fig. 1) (Almeida, 1983a, 1983b; Castro y Del
Papa, 2015). Para este trabajo, los restos humanos analizados corresponden a los sitios La Correntina, Cerro Lorenzo 2, Sambaquí de Puerto Landa y Túmulo I La Argentina (Tabla 1). La Correntina es un sitio dispuesto en depósitos arenosos (albardones), mientras que Cerro Lorenzo 2 y Sambaquí de Puerto Landa constituyen "cerritos"; este último fue considerado una construcción antrópica por Greslebin (1931) y como un basural sobre elevado por acumulación gradual de desechos por Krapovickas (1957). El Túmulo I La Argentina probablemente corresponda al sitio arqueológico estudiado por Aparicio (1928), quien postuló que el "cerro" de La Argentina es un cementerio, utilizado como vivienda sólo en caso de emergencia por la crecida de las aguas. Todos los restos humanos recuperados en este sitio corresponden a inhumaciones secundarias y aparecen entremezclados con los demás materiales arqueológicos (artefactos líticos, alfarería y restos faunísticos) (Castro y Del Papa, 2015).

Este conjunto osteológico, que se encontraba prácticamente inédito, fue estudiado a partir del año 2012 por Castro y Del Papa; como resultado de estas primeras aproximaciones se pudo establecer que la colección se encuentra representada por un número mínimo de individuos (NMI) de 80, habiendo tanto adultos masculinos y femeninos, como subadultos. Debido a la ausencia de fechados radiocarbónicos previos en esta porción del río Uruguay, las dataciones realizadas por Castro y Del Papa (2015) han sido una información fundamental. Estas ubican a los sitios arqueológicos en el Holoceno tardío, lo que confirma la presencia humana en este sector del Uruguay inferior entre ca. 2000-600 años AP (Tabla 1). Asimismo, en los restantes sitios presentados aquí y aún no datados, la presencia de alfarería, cuyas dataciones más antiguas en contextos arqueológicos de áreas circundantes se ubican en $c a .3000$ años AP (Politis, Martínez y Bonomo, 2001; Taddei, 1987), sugiere su ubicación temporal en el Holoceno tardío. La muestra utilizada en este trabajo está conformada por 11 individuos adultos de ambos sexos e incluyen a los tres individuos sobre los que se realizaron los fechados radiocarbónicos (Tabla 1).

\section{Análisis morfométricos y estadísticos}

Para los análisis morfométricos los cráneos se orientaron por el plano de Frankfurt y las imágenes digitales se obtuvieron en norma lateral (lado 
izquierdo) con una cámara digital Fuji Film S700. Se registraron las coordenadas para dos landmarks (घ): nasion (Buikstra y Ubelaker, 1994) y postmastoides (intersección del contorno del hueso occipital y la apófisis mastoides) y 32 semilandmarks (•) (Fig. 2). Las imágenes digitales de las muestras se procesaron con el software TPSUtil (Rohlf, 2006). Los puntos de referencia y las coordenadas semilandmarks se registraron por medio del software tpsDIG 2 (Rohlf, 2006).

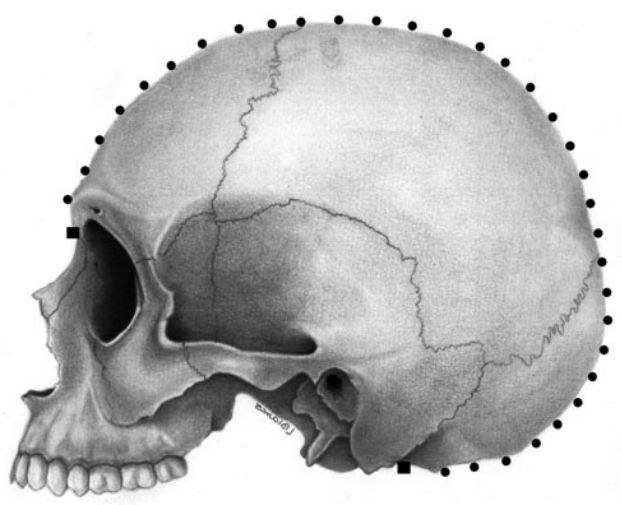

Fig. 2. Landmarks y semilandmarks registrados sobre imágenes digitales del cráneo obtenidas en norma lateral.

Los semilandmarks craneales en norma lateral fueron alineados utilizando el método de deslizamiento de semilandmarks propuesto por Bookstein y Green (Bookstein, 1997; Green, 1996). Esta operación amplía el Análisis de Procrustes Generalizado (GPA) (Rohlf, 1990; Rohlf y Slice, 1990) y además permite de forma óptima trasladar, escalar y rotar puntos de referencia. De esta manera los puntos semilandmark se deslizan a lo largo de la curva de contorno hasta que coincidan. Esto se hace porque los puntos individuales en las curvas no son, al parecer, necesariamente homólogos entre individuos. En consecuencia, la variación sobre las direcciones tangentes no es informativa, sólo la variación de la coordenada perpendicular al contorno conlleva información sobre las diferencias entre especímenes o grupos. (Bookstein, 1997; Bookstein, Streissguth, Sampson, Connor, y Barr, 2002). En este estudio, los semilandmarks se deslizan a lo largo de sus respectivas curvas con el fin de minimizar la distancia Procrustes entre el sujeto y una referencia (Bookstein, et al., 2002), utilizando el software Semiland6 (Sheets, 2003). El método de Relative warps (RW) se utilizó para comparar las configuraciones de los puntos de referencia y semilandmarks (Bookstein, 1991). Los RW son vectores de componentes principales de las deformaciones parciales, que son variables generadas por las transformaciones de los análisis Thin-Plate Spline (Bookstein, 1991), más los vectores de componentes uniformes. Los análisis de RW se realizan por medio del software PCAGEN6 (Rohlf, 2006). Un aspecto importante de estos análisis es que se puede expresar los resultados de los análisis estadísticos como una deformación de cada caso sobre la forma media o de referencia (Bookstein, 1991; Rohlf, 1998).

A fin de poder descartar diferentes fuentes de error se llevaron a cabo una serie de procedimientos. En primer lugar, se desarrolló un protocolo para la obtención de imágenes lo que permitirá registros similares para toda la muestra. En segundo término, el procesamiento y digitalización de landmarks y semilandmarks fueron realizados exclusivamente por uno de los autores (VAP). Esto permitió estimar el error interobservador. Por último, se llevó a cabo el cálculo del error intraobservador, para su puesta a punto se tomaron dos medidas repetidas a un subconjunto de individuos $(\mathrm{n}=13)$ donde se digitalizaron los landmarks y semilandmarks y sobre los que se realizó un análisis de ANOVA de medidas repetidas. Los cambios en la forma fueron representados con grillas de deformación a partir del uso de TPS-grids.

A fin de poder evaluar la existencia de diferencias significativas en la variación en forma de la bóveda craneana entre las muestras en estudios, se llevó a cabo un análisis de la varianza de una vía considerando un alfa $=0.05$, y empleando como método de comparación múltiple el test de Tukey para un alfa $=0.05$.

\section{RESULTADOS}

Debido a que las diferencias entre sexos de los componentes 1,2 y 3 no fueron significativas (Tabla 2) se prosiguió con el análisis sin diferenciar por sexo. En la Figura 3, se presentan los escores de los dos primeros RW de la totalidad de las muestras. Ambos explican el $72 \%$ de la variabilidad. El análisis de RW realizado sobre la forma de la bóveda aporta información sobre cuáles son las variables que más influyen en la expresión morfológica. En este caso se observa que la de- 
TABLA 2. Prueba de Anova para diferencias sexuales

\begin{tabular}{cccc}
\hline Población & $\mathrm{Cp}$ & $\mathrm{F}$ & $p$ \\
\hline \multirow{4}{*}{ Los Marinos } & $\mathrm{Cp} 1$ & 0.07 & 0.801 \\
& $\mathrm{Cp} 2$ & 3.55 & 0.075 \\
& $\mathrm{Cp} 3$ & 0.59 & 0.453 \\
& $\mathrm{Cp} 1$ & 0.41 & 0.525 \\
Delta inferior & $\mathrm{Cp} 2$ & 2.85 & 0.102 \\
& $\mathrm{Cp} 3$ & 0.13 & 0.721 \\
& $\mathrm{Cp} 1$ & 0.20 & 0.664 \\
& $\mathrm{Cp} 2$ & 1.31 & 0.289 \\
& $\mathrm{Cp} 3$ & 10.89 & $0.013 *$ \\
\hline
\end{tabular}

$* \mathrm{p}<0,05$ formación explicaría gran parte de la dispersión de las muestras en estudio sobre el RW1. Se observa una distribución continua de la variación en la morfología del cráneo. La muestra procedente del delta superior del río Paraná (DS) se posiciona en los valores negativos del primer y en menor medida del segundo RW. Esta muestra presenta una tendencia hacia la compresión en la región del lambda y expansión en la región del frontal (Fig. 3a). La muestra procedente del tramo inferior del rio Uruguay (CiRU) se posiciona en los valores positivos del primer y segundo RW. En este caso, la grilla de deformación muestra una tendencia hacia la compresión frontal y occipital (Fig. 3b). La muestra procedente del delta inferior del río Paraná (DI) se posiciona en el centro a

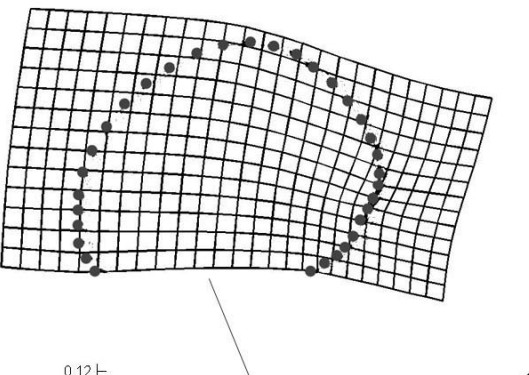

b

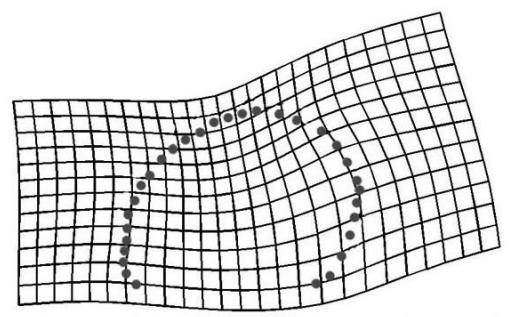

c.

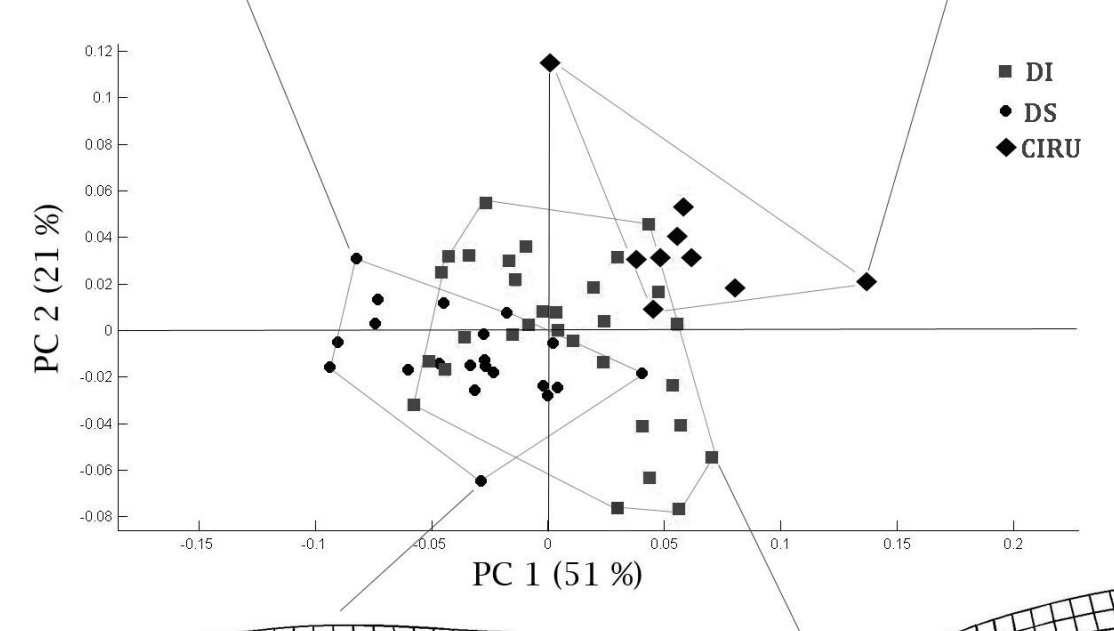

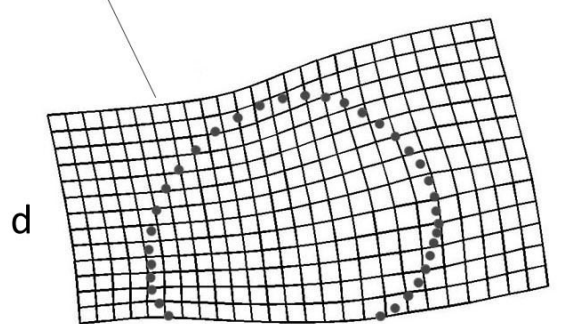

Fig. 3. Análisis de relative warp (RW) realizado a partir de las variables registradas en el cráneo en norma lateral procedentes de los sitios del Delta del río Paraná y cuenca inferior del río Uruguay. Las grillas representan a los cráneos con deformación tabular erecto plano lámbdica (a), circular (b), no deformados (c) y deformación circular leve (d). 
del cluster, tomando valores tanto positivos como negativos de ambos RW. Ésta presenta una dispersión mayor con superposición de las muestras anteriores. Los individuos que se identifican con bajo grado o ninguna deformación se posicionan en los valores más cercanos a cero de ambos RW.

Como resultado del ANOVA del RW1, cuya variación total explicada es del $51 \%$ (variable de deformación), se obtuvo un $\mathrm{F}=21,7100$, con un valor de $p$ menor a 0.0001 , lo cual indicó la existencia de diferencias significativas entre las muestras analizadas. El test de Tukey, para un alfa de 0.05 permitió establecer que las diferencias evidenciadas por el ANOVA se producen entre las tres muestras en estudio. Para el RW2, que explica un $21 \%$ de la variación, se un $\mathrm{F}=9,2300$, con un valor de $p$ menor a 0.0001 , lo cual permitió constatar la existencia de diferencias significativas entre las muestras analizadas. El test de Tukey, para un alfa de 0.05 permitió establecer que las diferencias evidencias por el ANOVA se producen entre dos grupos, por un lado las muestras correspondientes a DS y DI y por otro CiRU.

\section{DISCUSIÓN Y CONCLUSIONES}

El abordaje en torno al análisis de la variabilidad en la morfología presente en la bóveda del cráneo, a partir de las prácticas intencionales deformatorias, para el área en estudio, es un problema cuya naturaleza compleja es el producto de múltiples factores (e.g. aspectos ideológicos en la práctica deformatoria de la bóveda del cráneo, la ausencia de datos previos, la escasa cronología absoluta para la región o el número de muestras utilizadas para los análisis). Dicha complejidad introduce limitaciones a los objetivos propuestos, por lo que la identificación de los factores que la constituye será fundamental para lograr una interpretación adecuada.

Desde un punto de vista teórico entendemos que en el resultado final en la configuración fenotípica del cráneo intervienen una serie de efectos genéticos y ambientales, en donde identificamos entre estos últimos a las prácticas deformatorias como componente cultural, lo que estaría determinando particularidades en la morfología del cráneo adulto, resultando en una variación mayor de la esperada a diferentes niveles geográficos (Cocilovo y Varela, 2010). Debido a que en este trabajo se analizó la variabilidad morfológica de la bóveda craneana en individuos adultos, no fue posible controlar la influencia ambiental durante el desarrollo ontogenético de los mismos; esto implicó que no fuera posible de aislar los componentes ecofenotípicos y genéticos de la variación morfológica (Carroll, Hendry, Reznick, y Fox, 2007; Hendry y Kinnison, 1999). En este sentido, la variabilidad morfológica observada en las muestras analizadas da cuenta de las diferencias en las técnicas de deformación aplicadas por las poblaciones del delta superior del río Paraná, delta inferior del río Paraná y el tramo inferior del río Uruguay.

Mediante este abordaje se logró integrar los resultados obtenidos, a la discusión que se viene desarrollando desde finales del siglo XIX (Broca, 1879; Virchow, 1892) y todo el siglo XX (Baffi y Berón, 1992; Dembo y Imbelloni, 1938; Imbelloni, 1924-1925) sobre la distribución geográfica y cronológica de las deformaciones de la bóveda del cráneo, en el actual territorio de la República Argentina. Representando un aporte que puede ser incorporado al esquema general de investigaciones sistemáticas que se vienen realizando.

Se pudo establecer que para el área comprendida por el delta superior e inferior del río Paraná y tramo inferior del río Uruguay, existen al menos dos tendencias en el uso de prácticas deformatorias. Por un lado, se observa la existencia de deformaciones que comprometen la compresión del frontal y occipital (Fig. 3b), vinculadas cronológicamente con el Holoceno tardío inicial y asociados con los sitios del tramo inferior del río Uruguay (ver Tabla 1). Por otro lado, otra expresión deformatoria tendiente hacia la compresión del lambda y expansión del frontal (Fig. 3a), asociada cronológicamente con el Holoceno tardío final y vinculada fundamentalmente con el sitio arqueológico del delta superior del río Paraná. Por otro lado, la muestra proveniente de los sitios arqueológicos del delta Inferior del río Paraná, está asociada con todas las formas (a, b, c y d) representadas en la Figura 3, tendiendo a agruparse en torno al valor 0 del RW1.

De esta manera, se logra incorporar al contexto de las investigaciones sistemáticas, sobre la manifestación de las deformaciones artificiales realizadas en otras áreas del país, la dimensión de este fenómeno para el Delta del Paraná y cuenca inferior del río Uruguay. El esquema crono- 
lógico en la expresión morfológica de la bóveda craneana descripta para el área de estudio, mantiene parcialmente el patrón general propuesto por otros autores en áreas vecinas. Se establece, para el Holoceno tardío final, mediante fechados radiocarbónicos, deformaciones del tipo tabular erecto, en áreas tales como Pampa (Baffi y Berón, 1992), sudeste de Pampa (Barrientos, 2001), valle inferior del río Colorado (Martínez, Bayala, Flensborg, y López, 2006) y sur de Mendoza (Perez, 2006). Para áreas tales como el litoral atlántico del norte de Patagonia, el sudeste de la región Pampeana y la subregión Pampa seca (Baffi y Berón, 1992; Bernal et al., 2008; Madrid y Barrientos, 2000), la presencia de deformaciones con compresiones en el occipital y frontal posee una profundidad cronológica que se extiende hasta el Holoceno tardío inicial (Bernal et al., 2008), coincidiendo con el fechado de 2000 años AP dado a conocer para la cuenca inferior del río Uruguay (Castro y Del Papa, 2015).

Estas expresiones deformatorias están asociadas con las prácticas no intencionales y principalmente son la consecuencia del uso de tablillas o bandas para envolver la cabeza del bebé mientras es transportado en cunas (Brothwell, 1981), práctica frecuentemente utilizada por grupos de cazadores-recolectores con alta movilidad. Las diferentes expresiones que se observan tanto en la intensidad de la práctica deformatoria (e.g. Delta Superior y Delta Inferior) como en las particularidades morfológicas que adquiere la bóveda craneana en cada una de las áreas, puede ser entendida como el resultado de prácticas intencionales para la obtención de particularidades morfológicas, relacionadas con la necesidad de dar una forma específica, cuyo objetivo es el de ser usado como indicador de identidad social (Torres-Rouff, 2003). Este argumento parecería tomar validez para cada uno de los sectores ya que las investigaciones arqueológicas muestran la presencia de diferentes grupos culturales a través del Holoceno tardío.

Desde un punto de vista arqueológico, la ocupación del Delta del río Paraná y la cuenca inferior del río Uruguay, posee una cronología relativamente tardía (Holoceno tardío). Se han localizado sitios asociados con distintas geoformas, albardones, islas y la llanura adyacente. El material bioarqueológico procede de estas diferentes locaciones. Ambas áreas comparten la construc- ción de elevaciones en tierra (cerritos) como lugar de vivienda y la manufactura de la cerámica Goya-Malabrigo caracterizada por los apéndices zoomorfos, las campanas y la decoración geométrica de las vasijas (Politis y Bonomo, 2012).

En un plano interregional, se logra posicionar en un esquema general de relaciones entre prácticas deformatorias y cronologías absolutas a las poblaciones del Delta del río Paraná y de la cuenca inferior del río Uruguay, con otras regiones de la Argentina. Desde un plano intra-regional los resultados obtenidos, en torno a las formas de las prácticas deformatorias, permiten establecerla existencia de dos tendencias en la morfología, una asociada con deformatoria tendiente hacia la compresión del lambda y expansión del frontal y otra que permite observar una tendencia hacia la compresión del frontal y occipital. Sin embargo, cabe señalar que desde un punto de vista metodológico, la no disponibilidad de una muestra con mayor representatividad, que permita obtener resultados que reflejen con mayor rigor el rol que cumplen estas prácticas culturales en esta amplia región, no limitan la posibilidad de la realización del análisis de este fenómeno, aunque sí reduce su precisión.

\section{AGRADECIMIENTOS}

A la Dra. Livia Kozameh (UNR) por permitir el acceso a la muestra correspondiente a Cerro Grande de la Isla Los Marinos y al Señor Raúl Almeida por permitir el acceso a las colecciones del Museo Arqueológico Manuel Almeida (Gualeguaychú, Entre Ríos). A Laura Blanco (FCNyM-UNLP) quien realizó el dibujo del cráneo en norma lateral. A los evaluadores anónimos que ayudaron a mejorar el trabajo.

\section{LITERATURA CITADA}

Almeida, M. S.(1983a). De Soriano y su historia. Revista Histórica de Soriano, 25, 85-87.

Almeida, M. S. (1983b). El arte prehistórico. Bicentenario de Gualeguaychú. Homenaje de diario El Día al cumplir 200 años de su fundación. 1783 -18 de octubre- 1983

Aparicio, F. de. (1928). Notas para el estudio de la arqueología del Sur de Entre Ríos. Anales de la Facultad de Ciencias de la Educación 3, 1-63.

Baffi, E. I. y Berón, M. (1992). Los restos óseos humanos de Tapera Moreira (La Pampa), y la deformación artificial en la región pampeana. Análisis tentativo. $\mathrm{Pa}$ limpsesto. Revista de Arqueología, 1, 25-36. 
Barrientos, G. (2001). Una aproximación bioarqueológica al estudio del poblamiento prehispánico tardío del $\mathrm{Su}-$ deste de la Región Pampeana Intersecciones en Antropología, 2, 3-18.

Bernal, V. (2008). Procesos de diferenciación biológica entre poblaciones humanas del Holoceno tardio de Patagonia. Una aproximación desde la variación métrica dental. Tesis doctoral. Facultad de Ciencias Naturales y Museo, Universidad Nacional de La Plata, La Plata

Bernal, V., González, P., Pérez, S. I. y Pucciarelli, H. (2008). Entierros humanos del noreste de Patagonia: Nuevos fechados radiocarbónicos. Magallania, 36(2), 125-134. doi: 10.4067/S0718-22442008000200013

Berón, M. y Baffi, E. I. (2003). Procesos de cambio cultural en los cazadores recolectores de la provincia de La Pampa, Argentina. Intersecciones en Antropología, 4, 29-43.

Berón, M. y Luna, L. (2009). Distribución espacial y cronológica de la deformación craneana tabular erecta en Pampa y Norpatagonia. En F. Santiago, M. Salemme, M. Alvarez, E. Piana, M. Vazquez, M. E. Mansur (Comp.). Arqueología de la Patagonia: una mirada desde el último confin (pp. 561-575). Ushuaia, Argentina: Utopías

Bonomo, M. (2012). Historia prehispánica de Entre Ríos. Buenos Aires, Argentina: Fundación de Historia Natural "Félix de Azara".

Bonomo, M. (2013). Reanálisis de la colección de Samuel Lothrop procedente del Delta del Paraná. Relaciones de la Sociedad Argentina de Antropología, XXXVIII (1), 169-198.

Bonomo, M., Capdepont, I. y Matarrese, A. (2009). A1cances en el estudio de colecciones. Los materiales arqueológicos del Delta del río Paraná depositados en el Museo de La Plata (Argentina). Revista de Arqueología Sudamericana, 5, 68-101.

Bonomo, M., Politis, G. y Gianotti, C. G. (2011). Montículos, jerarquía social y horticultura en las sociedades indígenas del delta del Río Paraná (Argentina). Latin American Antiquity, 22(3), 297-333. doi: 10.7183/1045-6635.22.3.297

Bookstein, F. L. (1991). Morphometric tools for landmark data: geometry and biology. Cambridge, United Kingdom: Cambridge University Press. doi: 10.1002/ sim.4780120711

Bookstein, F. L. (1997). Landmark methods for forms without landmarks: localizing group differences in outline shape. Medical Image Analysis, 1, 225-243. doi: 10.1016/S1361-8415(97)85012-8

Bookstein, F. L., Streissguth, A. P., Sampson, P. D., Connor, P. D. y Barr, H. M. (2002). Corpus callosum shape and neuropsychological deficits in adult males with heavy fetal alcohol exposure. NeuroImage, 15 , 233-251. doi: 10.1006/nimg.2001.0977

Bórmida, M. (1950). Cementerios indígenas prehispánicos en la zona de la Laguna del Juncal. Anales del Museo Nahuel Huapi, 2, 101-108.

Bórmida, M. (1953-1954). Los antiguos Patagones. Estudio de craneología. Runa, VI, 55-96.

Blom, D. E. (1999). Tiwanaku regional interaction and social identity: abioarchaeological approach. (Tesis doctoral). University of Chicago, Chicago.

Broca, P. (1879). Sur un mode peu connu de deformation toulousaine. Bulletins de la Société d'anthropologie de Paris, 2(2), 699-701. doi: 10.3406/bmsap.1879.5268

Brothwell, D. R. (1981). Digging up bones. New York, USA: Cornell University Press. doi: 10.1002/ gea.3340010210
Buisktra JE, Ubelaker DH. 1994. Standards for data collection from human skeletal remains. Arkansas Archaeological Survey Research Series No. 44. Faytteville, USA. doi: 10.1002/ajhb.1310070519

Caggiano, M. A. (1983). Caracterización y antropodinamia prehispánica en el N.E. argentino a propósito de los primeros fechados radiocarbónicos para el Delta del Paraná. Relaciones de la Sociedad Argentina de Antropología, $X V, 61-76$.

Caggiano, M., Flores, O. B., Méndez, M. G. y Salceda, S. A. (1978). Nuevos aportes para el conocimiento antropológico del delta del Paraná. Relaciones de la Sociedad Argentina de Antropología, XII, 155-173.

Carroll, S. P., Hendry, A. P., Reznick, D. N. y Fox, C. W. (2007). Evolution on ecological time-scales. Functional Ecology, 21, 387-393. doi: 10.1111/j.13652435.2007.01289.x

Castro, J. C. y Del Papa, M. C. (2015). La estructura del registro bioarqueológico del río Uruguay inferior. Análisis de la colección osteológica humana del Museo Arqueológico Manuel Almeida (Gualeguaychú, Entre Ríos). Intersecciones en Antropología, 16, 195-205.

Cocilovo, J. A. y Varela, H. H. (2010). La distribución de la deformación artificial del cráneo en el Área Andina Centro Sur. Relaciones de la Sociedad Argentina de Antropología, XXXV, 41-68.

Cocilovo, J. A.,Varela, H. H. y O’Brien, T. G.(2010). Effects of artificial deformation on cranial morphogenesis in the South Central Andes. International Journal of Osteoarchaeology, 21 (3): 300-312. doi: 10.1002/oa.1141.

Della Negra, C., Novellino, P., Perez, S. I., Hajduk, A. y Bernal, V. (2008). Investigaciones arqueológicas y bioarqueológicas en el sitio Aquihueco (Norpatagonia): nuevos resultados. En: En F. Santiago, M. Salemme, M. Alvarez, E. Piana, M. Vazquez, M. E. Mansur (Comp.), Arqueología de Patagonia: una mirada desde el último confin. (pp. 669-676) Ushuaia, Argentina: Utopías.

Del Papa, M. C. y Perez, S.I. (2007). The influence of artificial cranial vault deformation on the expression of cranial nonmetric traits: its importance in the study of evolutionary relationships. American Journal of Physical Anthropology, 134(2), 251-262. doi: 10.1002/ ajpa. 20665

Dembo, A. e Imbelloni, J. (1938). Deformaciones intencionales del cráneo de carácter étnico. Buenos Aires, Argentina: J. Anesi.

D'Orbigny, A. [(1839) 1944]. El hombre americano considerado en sus aspectos fisiológicos y morales. Traducción de Alfredo Cepeda. Buenos Aires, Argentina: Editorial Futuro.

Drube, H. (2010). La deformación de cráneo en las sociedades precolombinas de Santiago del Estero. Relaciones de la Sociedad Argentina de Antropología, XXXV, 69- 84.

Fortich Baca, V. I. (1977). Acerca de algunos restos óseos humanos del nordeste argentino. Relaciones de la Sociedad Argentina de Antropología, XI, 91-100.

Frieß, M. y Baylac, M. (2003). Exploring artificial cranial deformation using Elliptic Fourier analysis of Procrustes aligned outlines. American Journal of Physical Anthropology, 122 (1), 11-22. doi: 10.1002/ajpa.10286.

Gaspary, F. (1950). Investigaciones arqueológicas y antropológicas en un cerrito de la Isla Los Marinos (Pcia. de Entre Ríos). Publicación del Instituto de Arqueología, Lingüistica y Folklore, 23, 3-66.

Green, W. D. K. (1996). The thin-plate spline and ima- 
ges with curving features. En: K. V. Mardia, C. A. Gill y, I. L. Dryden (Eds.) Proceedings in image fusion and shape variability techniques. (pp. 79-87). Leeds, United Kingdom: Leeds University Press. doi: 10.1186/1742-9994-3-15

Greslebin, H. R. (1931). La estructura de los túmulos indígenas prehispánicos del departamento de Gualeguaychú provincia de Entre Ríos, República Argentina. Revista de la Sociedad de Amigos de la Arqueología, $V, 5-51$.

Hendry, A. P. y Kinnison, M. T. (1999). The pace of modern life: measuring rates of contemporary microevolution. Evolution, 53, 1637-1653. doi: 10.2307/2640428

Imbelloni, J. (1924-1925). Deformaciones intencionales del cráneo en Sud América. Revista del Museo de La Plata, 28, 329-407

Imbelloni, J. (1933). América, cuartel general de las deformaciones. Actas del XXV Congreso Internacional de Americanistas 1, 59-68.

Konigsberg, L. W., Kohn, L. A. P. y Cheverud, J. M. (1993). Cranial deformation and nonmetric trait variation. American Journal of Physical Anthropology, 90(1), 35-48. doi: 10.1002/ajpa.1330900103

Kozameh, L. y Brunás, O. (2011). Paleopatología: Paget óseo en un resto prehispánico. Microscopía y datación. Actualizaciones en Osteología, 7 (2), 93-95.

Krapovickas, P. (1957). Excursión arqueológica a Rincón de Landa. Revista Geográfica Americana, 41 (245), 149-156.

Lista, R. [(1878) 1998]. Los cementerios y paraderos Minuanes de la provincia de Entre Ríos. Revista de Antropología, Tomo I. En Obras de Ramón Lista, Tomo I (1877- 1886) (pp. 35-38). Buenos Aires, Argentina: Editorial Confluencia.

Lothrop, S. K. (1932). Indians of the Paraná delta river. Annals of the New York Academy of Sciences, XXXIII, 77-232.

Madrid, P. y Barrientos, G. (2000). La estructura del registro arqueológico del sitio Laguna Tres Reyes 1 (provincia de Buenos Aires): nuevos datos para la interpretación del poblamiento humano del Sudeste de la Región Pampeana a inicios del Holoceno tardío. Relaciones de la Sociedad Argentina de Antropología, XXV, 179-206.

Martínez, G., Bayala, P., Flensborg, G. y López, R. (2006) Análisis preliminar de los entierros humanos del sitio Paso Alsina 1 (Partido de Patagones, Provincia de Buenos Aires). Intersecciones en Antropología, 7, 95-108.

Moreno, F. P. (1882). El origen del hombre sud-americano, razas y civilizaciones de este continente. Contribuciones al estudio de las colecciones del Museo Antropológico y Arqueológico. Conferencia de la Sociedad Científica Argentina. Conferencia del 12 de octubre de 1882. Buenos Aires, Argentina: Pablo Coni.

Outes, F. (1912). Cráneos indígenas del departamento de Gualeguaychú (Prov. de Entre Ríos). Anales de la Sociedad Cientifica Argentina, 73, 5-37.

Pérez, S. I. (2006). El poblamiento holocénico del sudeste de la región pampeana: un estudio de morfometría geométrica craneofacial. (Tesis doctoral). Facultad de Ciencias Naturales y Museo, Universidad Nacional de La Plata, La Plata.

Perez, S. I. (2007). Artificial cranial deformation in South America: A geometric morphometrics approximation. Journal of Archaeological Science, 34(10),1649-1658. doi: 10.1016/j.jas.2006.12.003

Politis, G. y Bonomo, M. (2012). La entidad arqueológica
Goya-Malabrigo (Ríos Paraná y Uruguay) y su filiación Arawak. Boletín de la Sociedade de Arqueologia Brasileira, 25(1), 10-46.

Politis, G., Martínez, G. y Bonomo, M. (2001). Alfarería temprana en sitios de Cazadores- Recolectores de la Región Pampeana (Argentina). Latin American Antiquity, 12 (2), 167-181. doi: 10.2307/972054

Ramos van Raap, M. A. y Bonomo, M. (2016). Nuevos estudios de la colección bioarqueológica de los sitios Arroyo Malo, El Cerrillo y Arroyo Sarandí (Delta del Paraná). Intersecciones en Antropología, 3, 71-82.

Richtsmeier, J. T., Cheverud, J. M. y Buikstra, J. E. (1984). The relationship between cranial metric and nonmetric traits in the rhesus macaques from Cayo Santiago. American Journal of Physical Anthropology, 64(3), 213- 222. doi: 10.1002/ajpa.1330640303

Rohlf, F. J. (1990). Morphometrics. Annual Review of Ecology and Systematic, 21, 299-316. doi: 10.1146/annurev.es.21.110190.001503

Rohlf, F. J. (1998). On application of geometric morphometrics to studies of ontogeny and phylogeny. Systematic Biology, 47, 147-158. doi: 10.1007/s11692-0099055-x

Rohlf, F. J. (2000). Statistical power comparisons among alternative morphometric methods. American Journal of Physical Anthropology, 111, 463-478.

Rohlf, F. J. (2006). Tps serie softwares. Recuperado de http//life.bio.sunysb.edu-/morph/.

Rohlf, F. J. y Marcus, L. (1993). A revolution in Morphometrics. Trends in Ecology \& Evolution, 8 (4), 129 132. doi: 10.1016/0169-5347(93)90024-J

Rohlf, F. J. y Slice, D. E. (1990). Extensions of the Procrustes method for the optimal superimposition of landmarks. Systematic Zoology, 39, 40-59. doi: $10.2307 / 2992207$

Sheets, H. D. (2003). IMP-Integrated Morphometrics Package. Department of Physics, Canisius College, New York, Buffalo.

Sober, E. (1980). Evolution, population thinking, and essentialism. Philosophy of Science, 47 (3), 350-383. doi: $10.1086 / 288942$

Taddei, A. (1987). Algunos aspectos de la arqueología prehistórica del Uruguay. En: L. Núñez y B. Meggers (Eds.) Estudios Atacameños Investigaciones paleoindias al sur de la línea ecuatorial (pp. 62-93). San Pedro de Atacama, Chile: Universidad del Norte.

Torres LM. (1911). Los primitivos habitantes Delta del Paraná. La Plata, Argentina. Universidad de La Plata, Biblioteca Centenaria.

Torres-Rouff, C. (2003).Shaping identity: cranial vault modification in the Pre-Columbian Andes. (Tesis doctoral). University of California, California.

Torres Rouff, C. y Yablonsky, L. T. (2005). Cranial vault modification as a cultural artifact: a comparison of the Eurasian steppes and the Andes. HOMO-Journal of Comparative Human Biology, 56, 1-16. doi: 10.1016/j. jchb.2004.09.001

Ubelaker, D. (1984). Human skeletal remains. Washington, USA: Taraxacum Press

Vignati, M. A. (1941). Censo óseo de paquetes funerarios de origen guaraní. Revista del Museo de La Plata, 2, $1-11$.

Virchow, R. (1892). Crania ethnica americana. Berlín, Alemania: A. Asher.

Zeballos, E. y Pico, P. (1878). Informe sobre el túmulo prehistórico de Campana. Anales de la Sociedad Cientifica Argentina, 6, 244-260. 